\title{
Twenty-Four-Hour ACTH and Cortisol Pulsatility in Depressed Women
}

\author{
Elizabeth A. Young, M.D, Nichole E. Carlson, M.S., and Morton B. Brown, Ph.D.
}

Increased plasma cortisol in patients with major depression is a well documented finding, although it is present in only $25-30 \%$ of subjects with major depression. However, ACTH and cortisol are secreted in a pulsatile manner, so it is unclear if increased ACTH secretion occurs in depression and if there are changes in the pulsatile components of ACTH secretion. Ten-minute sampling for ACTH and cortisol was performed for $24 \mathrm{hr}$ in 25 premenopausal depressed women, whose age and menstrual cycle day matched control women. As a group, the depressed women demonstrated a trend to increase cortisol secretion ( $p=$ 0.089). There was no difference in mean cortisol between the patient group as a whole $(8.36 \pm 2.9 \mu \mathrm{g} / \mathrm{dl})$ and those patients meeting criteria for atypical depression $(8.38 \pm 1.9$ $\mu \mathrm{g} / \mathrm{dl})$, but patients meeting criteria for endogenous showed increased cortisol (12.17 $\pm 4 \mu \mathrm{g} / \mathrm{dl})$ Mean ACTH was not significantly different between patients and controls. Pulse analyses revealed similar number of secretory events and similar amplitudes for cortisol secretory bursts in patients and controls. The baseline component area under the curve of cortisol secretion was increased at a trend level $(p=.064)$ in depressed patients, and the baseline AUC for ACTH was significantly increased in depressed patients $(p=.045)$. No differences were found in pulsatile components of ACTH secretion between patients and matched controls. Harmonic analyses indicated no significant differences between patients and controls on any detected rhythm for ACTH or cortisol. These data suggest that the pulsatile and circadian components of the HPA axis are normal in premenopausal depressed women and that only $24 \%$ of depressed women demonstrate hypercortisolemia.

[Neuropsychopharamcology 25:267-276, 2001] (C) 2001 American College of Neuropsychopharamcology. Published by Elsevier Science Inc.
KEY WORDS: Cortisol; Depression; HPA axis; Hormone pulsatility; Circadian rhythms; Ultradian rhythms

Dysregulation of the HPA axis is one of the most common findings present in individuals suffering from major depression (Carroll et al. 1976a, 1976b; Sachar et al. 1973). The prevalence of abnormalities is in part dependent upon the measure chosen and the subgroup of depressed patients. About $30 \%$ of patients with major de-

From the Department of Psychiatry and Mental Health Research Institute (EAY) and Department of Biostatistics (NEC, MBB), University of Michigan, Ann Arbor, MI

Address correspondence to: Elizabeth A. Young, University of Michigan, Mental Health Research Institute, 205 Zina Pitcher Place, Ann Arbor, MI 48109-0729. E-mail: eayoung@umich.edu

Received March 2, 2000; revised October 18, 2000; accepted October 31,2000 pression demonstrate hypercortisolemia, while $66 \%$ of melancholic depressed subjects show non-suppression of cortisol to dexamethasone (Carroll et al. 1981; Halbreich et al. 1985; Linkowski et al. 1985; Pfohl et al. 1985; Rubin et al. 1987). Studies have found an association between cortisol non-suppression to dexamethasone and baseline cortisol (Poland et al. 1987) suggesting that increased cortisol secretion is the primary defect responsible for resistance to dexamethasone feedback.

While many studies have focused upon cortisol, the examination of $24 \mathrm{hr}$ ACTH secretion is less frequent and more problematic (Deuschle et al. 1997; Linkowski et al. 1985; Mortola et al. 1987; Pfohl et al. 1985). Earlier studies used radioimmunoassay methodology that was neither as sensitive nor as specific for intact ACTH as the current two-site IRMA methodology. Furthermore, the majority of studies used a sampling frequency that 
was greater than the half-life of the hormone, so that increases in ACTH secretion could be missed. Given that it is possible that increased cortisol could result from increased sensitivity of the adrenal to ACTH, it is critical to establish that increased cortisol secretion is reflecting a central CRH dysregulation rather than occurring as a consequence of changes in the adrenal gland.

In addition to mean hormone levels, the central nervous system controls a number of other hormone rhythms that include pulsatile rhythms and circadian and ultradian rhythms. In depressed patients, abnormalities in circadian regulation of the HPA axis have been proposed. An earlier onset of cortisol secretory episodes was first reported by Sachar et al. (1973), and phase advance of the cortisol rhythm has been observed in aging (Van Cauter et al. 1996). Large scale studies by Halbreich et al. (1985), Rubin et al. (1987), and Pfohl et al. (1985) were mixed in support of an earlier onset of the first cortisol secretory episode and cosinor analysis did not support a phase advance in the circadian rhythm of cortisol in any of these studies. A recent meta-analysis by Van Cauter of the cortisol data from Rubin et al. (1987), Sachar et al. (1973), Mortola et al. (1987), and others revealed evidence of overall increased glucocorticoid secretion with the largest effect at the nadir of the circadian rhythm (Wirz-Justice 1994). Furthermore, this analysis found evidence of an earlier onset of the first cortisol secretory episode in depression. Data from our group examining circadian variation in the response to the administration of metyrapone, a compound which blocks cortisol synthesis, revealed increased central/pituitary drive in the evening compared to the morning in patients with depression, supporting alterations in circadian driven HPA axis secretion (Young et al. 1994, 1997).

Hormone pulsatility is another critical aspect of endocrine secretion. In the HPG axis, pulse frequency is a critical factor beyond mean hormone levels in regulating the axis. GnRH pulse frequency regulates not only secretory patterns of LH and FSH, but also differentially regulates mRNA levels of the subunits of $\mathrm{LH}$ and FSH in the pituitary (Marshall et al. 1991). Furthermore, $\mathrm{GnRH}$ pulse frequencies that are too fast or too slow lead to infertility (Belchetz et al. 1987). It is known that cortisol and ACTH are secreted in pulses. However, much less is known about pulse frequency in the HPA axis than in the HPG axis and still less is known about depression. The report of Mortola et al. (1987) examined six patients and reported an increase in ACTH pulse frequency. However, the number of pulses of ACTH found by Mortola et al. (1987), 9.9 pulses in $24 \mathrm{hr}$ in controls, using Cluster to detect ACTH pulses is significantly less than the number found by Veldhuis and colleagues (40 pulses) in normal men using the newer deconvolution analysis (Veldhuis and Johnson 1992, 1986; Iranmanesh et al. 1990). Finally, while HPA axis overactivity in depression is the usual type of HPA axis dysregulation that is observed, Gold and colleagues have proposed, based upon data from patients with chronic fatigue syndrome and fibromyalgia, that patients with atypical depression manifest a hypoactive HPA axis and an underactive CRH neuron in the paraventricular nucleus (Crofford et al. 1994; Demitrack et al. 1991; Tsigos and Chrosous 1994). However, they have never examined HPA axis activity in depressed patients with atypical depression. To determine if any of the CNS controlled HPA axis rhythms, particularly pulsatile, circadian, or ultradian rhythms, differed between depressed patients and matched controls, the current studies were undertaken evaluating ACTH and cortisol pulsatility in 25 women with major depression and a control group of 25 women matched for age and menstrual cycle day.

\section{METHODS}

\section{Subjects}

All subjects were premenopausal women who ranged in age from 20-50 years. Depressed women were recruited from patients presenting to the University of Michigan Mood Disorders program for treatment. All studies were approved by the University of Michigan Institutional Review Board (IRB). All subjects were medically healthy and untreated for the current episode at the time of the study. All were free of psychotropics and any other medications except for non-prescription pain medications, for more than three months. No subject engaged in shift work or traveled across more than 3 time zones within the 3 months prior to study. Subjects were studied on the general medical Clinical Research Center (CRC), where they were admitted for $26 \mathrm{hr}$. All subjects signed an informed consent. The Structured Clinical Interview for DSM-IV (SCID-IV) and structured Hamilton rating was conducted on all patients by our research nurse to confirm the diagnosis. The clinical diagnosis of major depression agree with the SCID-IV diagnosis in all cases. Normal controls had no previous psychiatric diagnosis as confirmed by SCID non patient version (SCID-NP), conducted by our research nurse. They had no first degree relative with any Axis I disorder and no second degree relative with an anxiety or mood disorder as ascertained by questioning the subject on all relatives. All subjects underwent a screening physical exam, blood work and urine drug screen. All controls were individually matched to each patient and matched on age and menstrual cycle day and length.

\section{Protocol}

Subjects presented to the CRC at 8:00 A.M. on day 1, when an intravenous catheter was inserted. After a 60- 
min recovery period, 10 min sampling was initiated for $24 \mathrm{hr}$. Normal saline was infused throughout the study. While subjects were able to get up to go to the bathroom, they remained at bedrest for the $24 \mathrm{hr}$ of the study. All meals were standardized in time and content. Eating between meals was not permitted, but decaffeinated beverages were provided at set times. Subjects were free to turn off the lights at their usual sleep times, but all lights were off by midnight. During overnight sampling, blood was drawn with the aid of a flashlight and nurses recorded whether the subjects were awake or asleep with each $10 \mathrm{~min}$ sample. All samples were drawn in a plastic syringe and mixed with EDTA in a polypropylene tube. Samples were immediately placed on ice and spun at least every two hours. Previous studies in our laboratory have validated the stability of ACTH and cortisol when samples remained on ice for four hours. A $24 \mathrm{hr}$ urine was collected simultaneously during the $24 \mathrm{hr}$ stay on the CRC.

\section{Hormone Assays}

ACTH was assayed with Allegro HS ACTH IRMA (Nichols Diagnostics, San Juan Capistrano, CA, USA), a two-site assay highly specific for intact ACTH. The sensitivity of the assay is $5 \mathrm{pg} / \mathrm{mL}$. Intra-assay variability averaged $9 \%$, and interassay variability of the internal control sample averaged 19\%. Cortisol was assayed with DPC Coat-a-Count assay, a solid phase radioimmunoassay. An aliquot of the 24-hr urine was extracted prior to assay following the manufacturer's directions for assay of urinary cortisol. The detection limit of the assay is $0.2 \mu \mathrm{g} / \mathrm{dl}$. Intraassay variability averaged $5 \%$ and interassay variability of the internal control sample averaged $8 \%$. All samples collected were assayed for ACTH first, and then cortisol, to avoid loss of ACTH secondary to freezing and thawing. Each patient's samples were assayed with her matching control in both assays. Each assay measured only one patient and her matched control (290 samples total).

\section{Pulsatility and Statistical Analyses}

All ACTH and cortisol data were analyzed with Pulsefit and deconvolution (Kushler and Brown 1991; Veldhuis and Johnson 1992). However, because of the circadian rhythm of these hormones, the assumption that all secretion was the result of pulsatile mode led to unreasonably long half-lives for ACTH and cortisol, resulting in poor fit of the calculated pulses to the actual data. Thus the data were analyzed with a new algorithm, Smoothing Baseline Pulse Pulses (SBPP) (Guo et al. 1999), which allows for a changing baseline. This algorithm identifies every time point at which there is significant secretion, called inputs or secretory episodes. Adjacent secretory episodes are not combined into pulses and therefore the number of secretory episodes should exceed the number of pulses. The fitted parameters from the analyses consist of average baseline, number of secretory episodes, average amplitude, and half life. In addition, AUC was calculated for each hormone. Furthermore, the total hormone secretion was divided into two components: baseline and pulsatile. All pulsatile components were analyzed after subtraction of the baseline component. AUC, half-life, total secretion, average amplitude, and average baseline were log (base e) transformed and number of secretory episodes was square root transformed prior to analyses with a paired t-test. Results that show a marginal level of significance $(p<0.1)$ or better are reported.

To determine circadian and ultradian rhythms of cortisol and ACTH for the controls, harmonic analysis was used whereby both sine and cosine waves were fitted to the $24 \mathrm{hr}$ ACTH and cortisol values using the harmonics of $24 \mathrm{hr}$ (i.e., $24 \mathrm{hr}, 12 \mathrm{hr}, 8 \mathrm{hr}, 6 \mathrm{hr}, 3 \mathrm{hr}, 2 \mathrm{hr}, 1 \mathrm{hr}$, and 30 $\mathrm{min}$ ). If either sine or cosine term for a specific frequency was significant, both terms for that harmonic period remained in the model. The data for each individual, both patient and control, were then fitted by the circadian model identified above. Patient and control pairs were analyzed by paired $t$-test for differences in maximum time and $\log$ (base e) transformed amplitude (i.e., fitted maximum - minimum). In addition, a RM-ANOVA was used to compare patients and controls for each hormone, where blocks of time was the repeated factor.

\section{RESULTS}

All depressed subjects met DSM-IV criteria for major depression. Twenty-five depressed patients and twenty-five matched controls were studied. Six depressed subjects met criteria for definite endogenous by Research Diagnostic Criteria (RDC), seven met criteria for non-endogenous, and the remaining 12 subjects met RDC criteria for probable endogenous. By DSM-IV criteria, seven met criteria for atypical depression. Five of these were non-endogenous by RDC criteria. Seventeen patients were recurrent unipolar, seven patients were in their first episode of depression and one was bipolar II, depressed. Seven subjects had dysthymia preceding the onset of their current depressive episode; nine of the subjects met criteria for comorbid anxiety disorders $(\mathrm{GAD}=2$; panic disorder $=4$; simple phobia $=5$; social phobia $=2$ ). The mean age of the patients was $29.0 \pm$ 7.8 (SD). The mean age of the controls was $29.0 \pm 7.9$ (SD). The mean severity of depression as assessed by the Hamilton Depression Rating Scale (21 item) was $19.6 \pm 5.3(\mathrm{SD})$. The mean Hamilton score in the controls was $1.0 \pm 1.5(\mathrm{SD})$. Subjects were studied at random phases of the menstrual cycle; and the controls were matched for menstrual cycle day (follicular $n=$ 12; luteal $n=13$ ). 


\section{Mean cortisol and ACTH}

The mean cortisol data are shown in Figure 1. As can be seen, the depressed patients as a whole demonstrate a trend to increased cortisol secretion [mean cortisol AUC was 10,712 $\pm 3,611(\mathrm{SD})$ in controls and 12,051 $\pm 4,023$ (SD) in patients $(\mathrm{p}=.089)]$. The mean 24 -hr cortisol in controls was $7.25 \pm 1.5$ (SD) $\mu \mathrm{g} / \mathrm{dl}$. In depressed patients, the mean cortisol was $8.36 \pm 2.9$ (SD). Six of the 25 depressed patients demonstrated mean cortisol greater than the mean plus one SD of the control subjects $(9.7 \mu \mathrm{g} / \mathrm{dl})$.

Patients were divided by subtype into RDC-definite endogenous and DSM-IV atypical depression. The cortisol profiles for RDC endogenous depressed patients and the control women are shown in Figure 2. As a group, the increase in cortisol secretion is greater in the endogenous subgroup than the depressed population as a whole (compare Figures 1 and 2) with mean cortisol in endogenously depressed patients of $12.17 \pm 4.0$ $\mu \mathrm{l} / \mathrm{dl}$. However, even with the analysis restricted to the endogenous subgroup only, the cortisol differences are not significant. The mean cortisol value in patients meeting RDC criteria for non-endogenous is $8.7 \pm 1.9$, still elevated in comparison to control subjects, although not significantly. The cortisol profile for patients meeting DSM-IV atypical depression criteria and controls is shown in Figure 3. As can be observed, the cortisol levels were normal in those subjects. The mean cortisol in atypical depressed patients was $8.38 \pm 1.9$ (SD), the same as the mean for the entire depressed population (8.36). Of the six patients with cortisol greater than one SD above the mean, two met RDC criteria for definite endogenous, three for non-endogenous and one for probable endogenous.

We also examined the relationship between depression severity as assessed by the Hamilton (range 11-33) and mean cortisol secretion and there was no significant relationship $(\mathrm{F}=2.46, \mathrm{r}=0.11, \mathrm{p}=0.13)$. The mean 24-hr urinary free cortisol was $72 \pm 10$ in controls and $77 \pm 13$ in depressed women. There was no relationship $(\mathrm{r}=.10)$ between urinary free cortisol and 24$\mathrm{hr}$ mean plasma cortisol.

The 24-hr ACTH profiles for depressed and control women are shown in Figure 4. As can be observed, there is a small increase in plasma ACTH in women with major depression, although this increase is again not significant. The 24-hr mean ACTH in the controls was $3.5 \pm 0.23 \mathrm{pM}$ and in the depressed patients was $3.8 \pm 0.32 \mathrm{pM}$, or a $9 \%$ difference. This is only slightly smaller than the $15 \%$ increase seen in mean cortisol. The ACTH AUC was 5,635 $\pm 2,104$ (SD) in patients and $4,950 \pm 1,875(\mathrm{SD})$ in controls, a non-significant in-

\section{Mean Cortisol Profile in Depressed Patients and Matched Controls $(n=26)$}

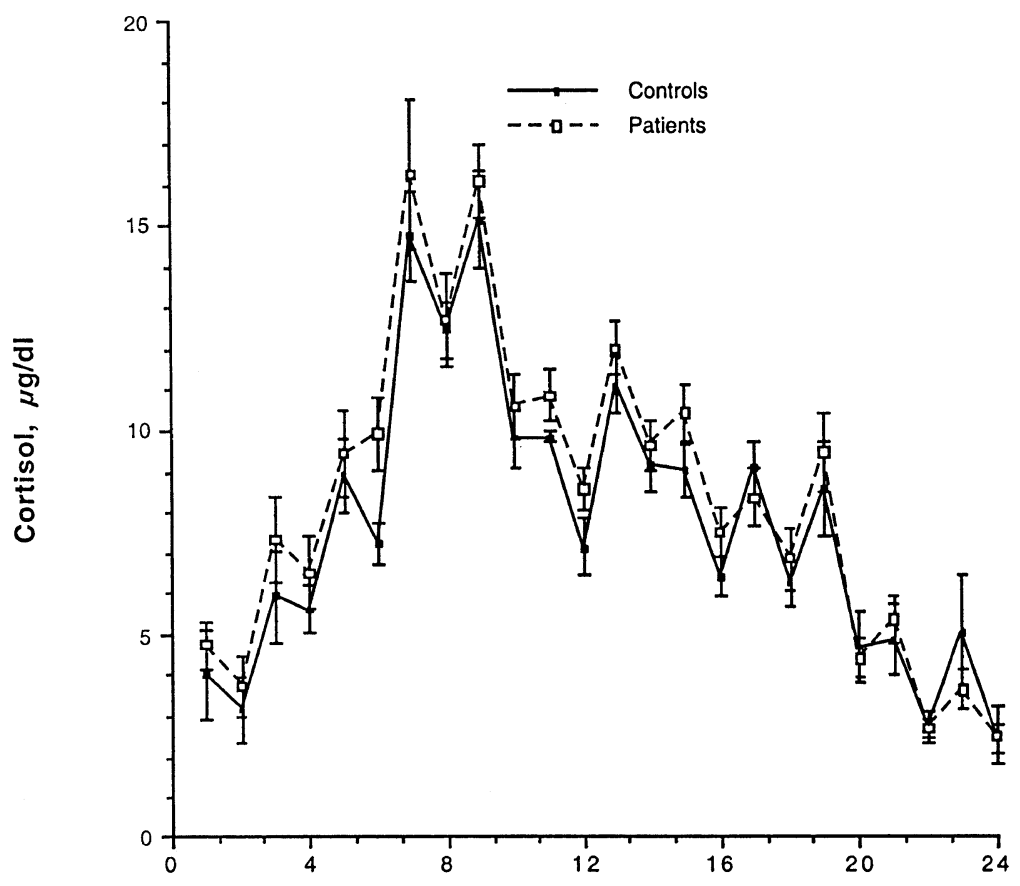

Figure 1. Twenty-four-hour cortisol profile in 26 depressed women and 26 age matched control subjects. Data are shown as the mean of 1-hr blocks (6 samples) across subjects. Although the cortisol levels appear higher in depressed patients, this showed only a trend level of significance. 
Cortisol Profile of Patients with RDC Endogenous Depression Compared to Control Subjects

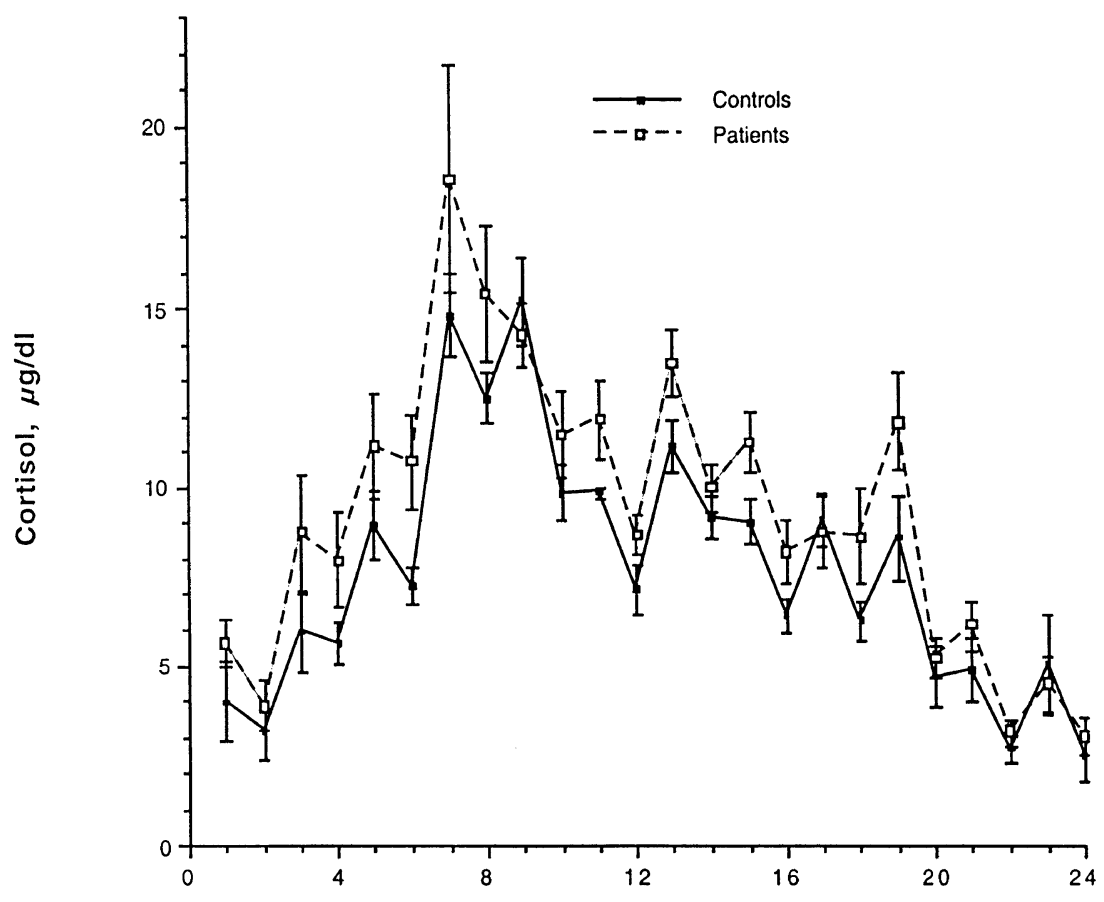

Figure 2. Cortisol profile in the 6 individuals who met RDC criteria for definite endogenous. Data are shown as the mean of 1-hr blocks (6 samples) across subjects. As expected, the cortisol levels appear higher although the difference is not significant.

Clock Time, 24H

crease. The mean ACTH in the endogenous subjects was $3.48 \pm 0.4 \mathrm{pM}$, again not different than controls. The mean ACTH in the six hypercortisolemic depressed women was $4.9 \pm 0.3$ (40\% increase), which was not significant $(\mathrm{p}=0.13)$, possibly due to the small sample size. The ratio of mean 24-hr cortisol to mean 24-hr ACTH was $2.2 \pm 0.19$ (SEM) in controls and $2.5 \pm$ 0.25 (SEM) in depressed patients, not significantly different. In the six subjects demonstrating cortisol one SD above the mean, the cortisol to ACTH ratio was $3.12 \pm$ 0.73 (SEM), not significantly different than controls.

\section{ACTH and Cortisol Pulsatility Analyses}

Table 1 presents the data for ACTH, and Table 2 for cortisol SBPP analyses. As can be seen, there are no significant differences between patients and controls in the number of secretory episodes, half-life of hormone secretion in either ACTH or cortisol. However, average baseline ACTH was significantly increased in depressed subjects $(\mathrm{p}=.041)$. Furthermore, there is large variability between individuals in these parameters in both patients and controls. For ACTH, approximately $60 \%$ of the ACTH secretion was in the baseline component and $40 \%$ in the pulsatile mode (Table 1). For cortisol, approximately $35 \%$ of the secretion was in the base- line mode, and $65 \%$ in pulsatile mode (Table 2). For both ACTH and cortisol, the observed increases in total AUC appear to be in the baseline component of secretion. In the case of cortisol, there was a trend $(p=.064)$ to increased cortisol in the baseline AUC, and in the case of ACTH the increase in baseline AUC was significant $(p=.045)$. The same analyses were conducted in the subgroup of depressed patients with increased baseline cortisol, but there were no significant differences in ACTH or cortisol pulse parameters in this group.

\section{Circadian Analyses}

To determine whether there were alterations in the quiescent period onset and offset of cortisol secretion, we adapted an algorithm from Van Cauter et al. (1996). Quiescent onset was defined as six consecutive samples (one hr) below $5 \mu \mathrm{g} / \mathrm{dl}$ and quiescent offset as six consecutive samples above $5 \mu \mathrm{g} / \mathrm{dl}$. The normal control and depressed patients demonstrate similar onset and offset (Table 3). The mean length of the quiescent period in controls was $7 \mathrm{hr} 16 \mathrm{~min} \pm 21 \mathrm{~min}$ (SEM) and in patients was $6 \mathrm{hr} 57 \mathrm{~min} \pm 29 \mathrm{~min}(\mathrm{SEM})$, a non-significant difference. Others have defined the onset of circadian activity as the time of the first secretory episode. However, because we drew more frequent samples 
Cortisol Profile of Patients with Atypical

Depression Compared to Control Subjects

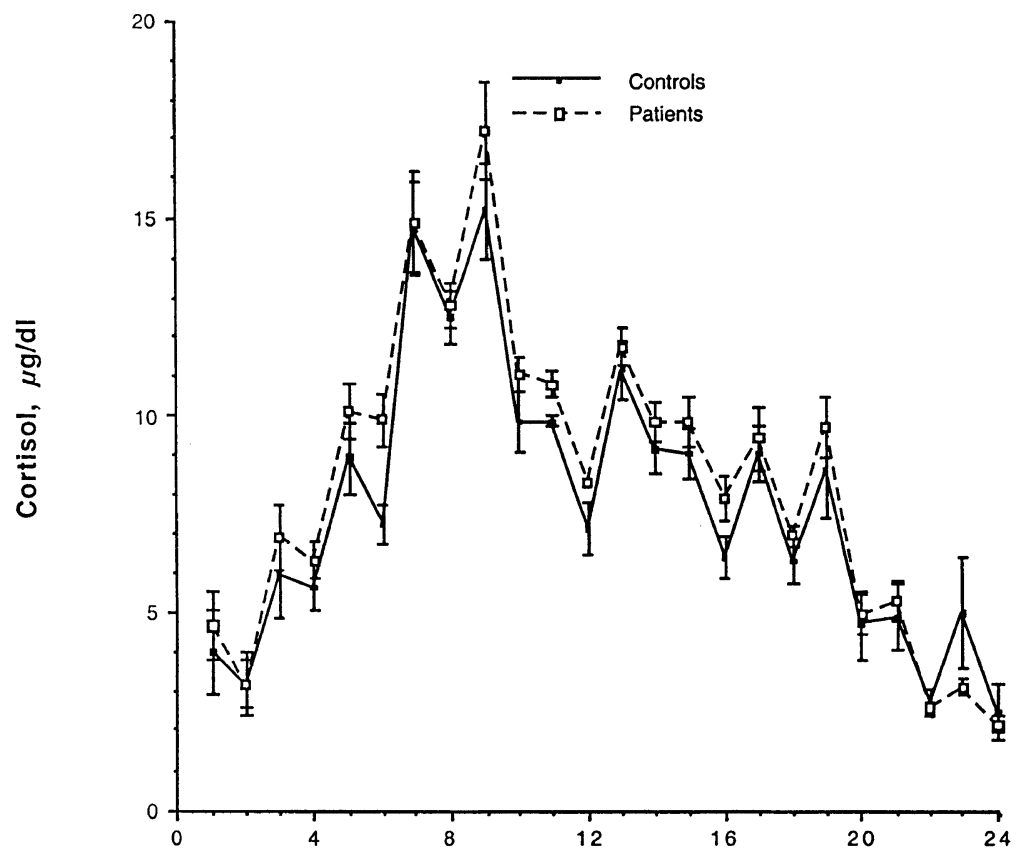

Figure 3. Cortisol profile in 6 patients who met criteria for atypical depression. Data are shown as the mean of 1-hr blocks (6 samples) across subjects. The cortisol profile looks the same as the depressed population as a whole, with no evidence of low cortisol.

Clock Time, 24H

than previous investigators, we were better able to define the nature of the pulsatile secretion of ACTH and cortisol. In each individual subject there was no quiescent time in terms of absence of cortisol pulses. Even in the late evening and at the time of sleep onset, pulsatile cortisol secretion continued to occur. For ACTH quiescent onset and offset, we used the definition of Linkowski et al. (1985), with the 24-hr mean hormone concentration used as a limit. Quiescent period onset was defined as six consecutive samples below the limit and quiescent offset as six consecutive samples above the limit. Again, no significant differences were found between patients and controls, including the subgroup of depressed patients with hypercortisolemia.

We examined the circadian pattern of hormone secretion by analyzing the data in four hour blocks. The four time blocks were: 22:00-1:50; 02:00-5:50; 06:0009:50; 10:00-13.50; 14:00-17:50; and 18:00-21:50. There was a significant effect of time $(p=0.0001)$ on ACTH levels, but no significant difference between patients and controls. Similarly for cortisol, there was a significant difference in cortisol levels over time $(p=0.0001)$ and marginal differences between patients and controls $(p=0.072)$. No 4-hr block comparison differed between patients and controls.

Based on harmonic analysis, for $\mathrm{ACTH}$, significant periods of $24 \mathrm{hr}, 8 \mathrm{hr}$, and $6 \mathrm{hr}$ were found. For cortisol, significant periods of $24 \mathrm{hr}, 12 \mathrm{hr}, 8 \mathrm{hr}$, and $2 \mathrm{hr}$ were found. For the 2-hr rhythm, we further evaluated if periods between 100 and $140 \mathrm{~min}$ fit the data better than $120 \mathrm{~min}$, but found the best fit with $120 \mathrm{~min}$. There were no significant differences between patient and controls on these parameters, nor was there a significant interaction with time. The data for clock time of maximum hormone secretion and amplitude are presented in Table 4 .

\section{Cross Correlational Analyses}

Cross correlational analysis between ACTH and cortisol was performed on the "pulsatile" component of the ACTH and cortisol secretion (baseline subtracted). The maximum correlation was found at $-10 \mathrm{~min}$ (i.e., ACTH leads cortisol by one sample) $(\mathrm{p}=.0001)$. The median lag was $-10 \mathrm{~min}$, and the mean $-13.1 \mathrm{~min}$. In 42 of 50 individuals, ACTH preceded cortisol, in five cases cortisol and ACTH rises were simultaneous, and in three individuals cortisol rises preceded ACTH. There were no patient control differences in these parameters.

\section{DISCUSSION}

The data presented in this study examine HPA axis dysregulation in a sub-group of the depressed patient 


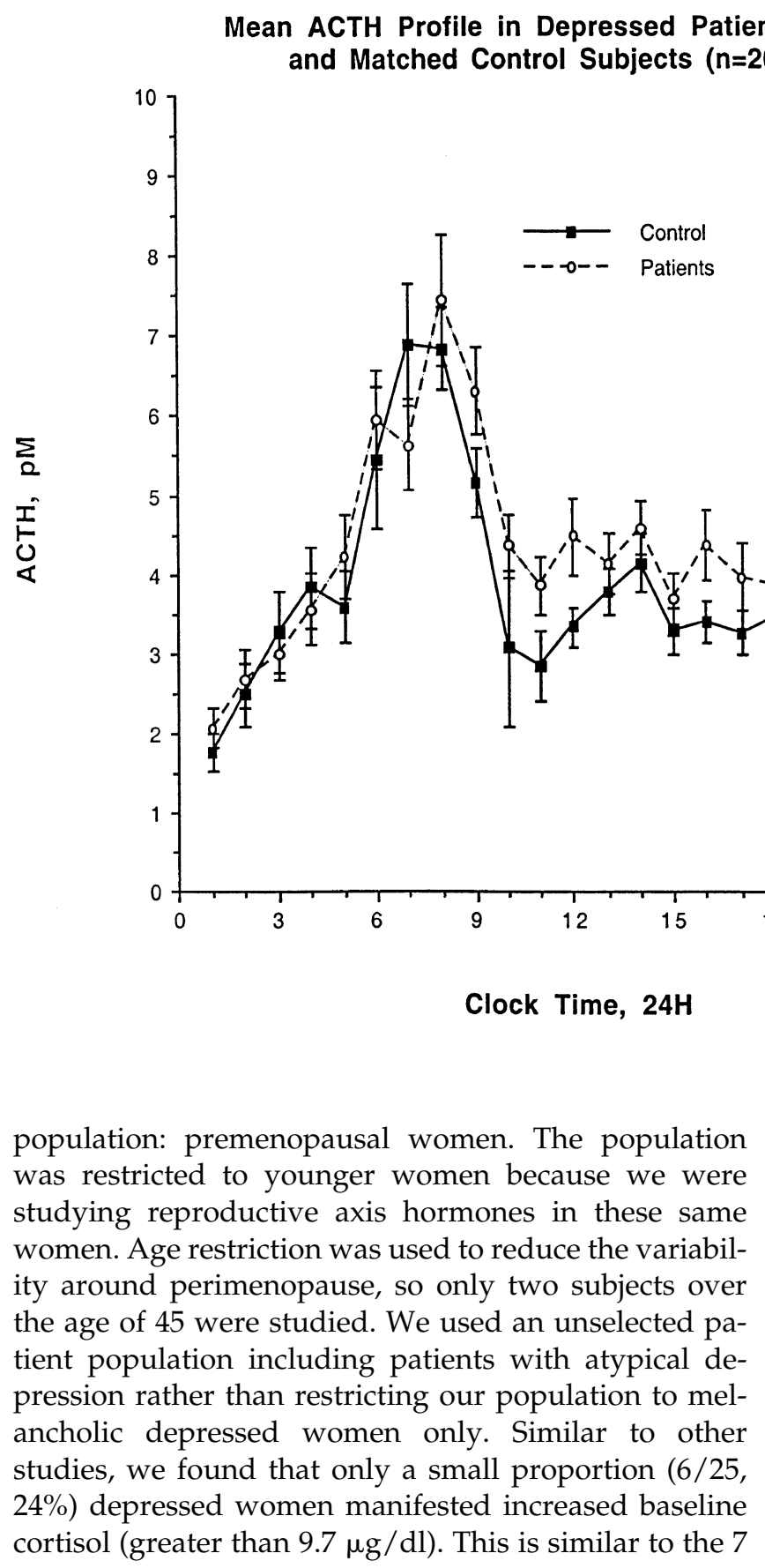

Table 1. Data from SBPP Analysis for ACTH

\begin{tabular}{lcc}
\hline & \multicolumn{2}{c}{ ACTH (Mean \pm SD) } \\
\cline { 2 - 3 } & Controls & Patients \\
\hline Number of secretory episodes & $23.2 \pm 11.6$ & $26.0 \pm 13.1$ \\
Average amplitude $(\mathrm{pM})$ & $2.2 \pm 1.5$ & $2.0 \pm 0.9$ \\
Average baseline $(\mathrm{pM})$ & $2.0 \pm 0.8$ & $2.5 \pm 1.0^{*}$ \\
Half-life (Minutes) & $23.2 \pm 10.8$ & $21.6 \pm 8.2$ \\
AUC baseline & $2945 \pm 1122$ & $3583 \pm 1356^{*}$ \\
AUC pulses & $2022 \pm 1405$ & $2032 \pm 1069$ \\
\hline
\end{tabular}

${ }^{*} p<.05$. of $32(22 \%)$ patients who had mean cortisol levels greater than $10 \mu \mathrm{g} / \mathrm{dl}$ in the study of Halbreich et al. (1985). The studies of Rubin et al. (1987) and Pfohl et al. (1985) used dexamethasone non-suppression to define subgroups and found normal baseline cortisol in the dexamethasone suppressors but significantly increased cortisol secretion in dexamethasone non-suppressors, which were $15 / 40$ (38\%) patients in the study by Rubin et al. (1987) and 8/25 (32\%) in the study by Pfohl et al. (1985). We did not use dexamethasone to select a subgroup, but overall our data in women agree with those studies including males and older women subjects

Table 2. Data from SBPP Analysis for Cortisol

\begin{tabular}{lcc}
\hline & \multicolumn{2}{c}{ Cortisol (Mean \pm SD) } \\
\cline { 2 - 3 } & Controls & Patients \\
\hline Number of secretory episodes & $38 \pm 12.8$ & $39.2 \pm 13.7$ \\
Average amplitude $(\mu \mathrm{g} / \mathrm{dl})$ & $2.9 \pm 1.6$ & $3.0 \pm 1.3$ \\
Average baseline $(\mu \mathrm{g} / \mathrm{dl})$ & $2.4 \pm 1.8$ & $3.0 \pm 1.6$ \\
Half-life $(\mathrm{min})$ & $49.3 \pm 42.5$ & $39.9 \pm 16.5$ \\
AUC baseline & $3353 \pm 2541$ & $4350 \pm 2239^{*}$ \\
AUC pulses & $7313 \pm 3878$ & $7534 \pm 3449$ \\
\hline${ }^{*} p<.064$. & &
\end{tabular}


Table 3. ACTH and Cortisol Quiescent Period Onset and Offset

\begin{tabular}{lrr}
\hline & \multicolumn{1}{c}{ Control } & \multicolumn{1}{c}{ Depressed } \\
\hline Cortisol quiescent onset & $19: 10 \pm 24 \mathrm{~min}$ & $19: 50 \pm 20 \mathrm{~min}$ \\
Cortisol quiescent offset & $2: 55 \pm 24 \mathrm{~min}$ & $2: 50 \pm 20 \mathrm{~min}$ \\
ACTH quiescent onset & $17: 05 \pm 33 \mathrm{~min}$ & $17: 06 \pm 26 \mathrm{~min}$ \\
ACTH quiescent offset & $4: 15 \pm 16 \mathrm{~min}$ & $4: 24 \pm 20 \mathrm{~min}$ \\
\hline
\end{tabular}

showing normal cortisol secretion in most depressed subjects. Interestingly, two studies have examined 24$\mathrm{hr}$ cortisol secretion in a male-only population and matched controls (Deuschle et al. 1997; Linkowski et al. 1985) and found significantly increased cortisol secretion. Thus, male depressed patients appear to demonstrate more reliable cortisol hypersecretion, raising the issue of sex differences in the HPA axis in depression. Furthermore, as clearly demonstrated by a recent metaanalysis, there are significant age effects on cortisol secretion in older women and we have eliminated older women from our study population (Van Cauter et al. 1996). We have previously observed significant age and gender effects in dexamethasone feedback on both pituitary and cortisol secretion, with low rates of dexamethasone non suppression in premenopausal women (Akil et al. 1993; Young et al. 1993).

Given that we were able to demonstrate increased cortisol in some of the depressed patients, the question of the relationship to diagnostic subtype was next addressed. Examining both melancholic and atypical depressed women, we saw evidence of normal-to-increased baseline cortisol compared to control women. The failure to detect significant differences in cortisol in both subgroups is probably secondary to the small sample size of these subgroups $(n=6$ for endogenous and $n=7$ for atypical depression). Of the six women with mean cortisol secretion greater than $9.7 \mu \mathrm{g} / \mathrm{dl}$, only two met RDC criteria for definite endogenous. Thus, there was no clear association with diagnostic subtype. Even subjects with atypical depression appeared to demonstrate normal to increased cortisol secretion. This is in contrast to the hypothesis of Tsigos and Chrosous (1994) who proposed low cortisol in this subgroup of patients.

The relationship of cortisol hypersecretion to ACTH secretion was also addressed. Since some but not all studies have demonstrated increased adrenal sensitivity to ACTH, it is unclear whether the hypercortisolemia of depression is a result of increased ACTH secretion (Krishnan et al. 1990). Previous studies on ACTH secretion were compromised by infrequent sampling, lack of drug free status, lack of specificity of the assay for intact ACTH, assays that were insufficiently sensitive, or sampling only at a restricted circadian phase. While some studies suggest increased mean ACTH (Deuschle et al. 1997, Pfohl et al. 1985), others found normal mean ACTH (Linkowski et al. 1985, Mortola et al. 1987). Our data show no significant differences but the mean is slightly elevated $(9 \%)$ in depressed women. The six patients with increased baseline cortisol also demonstrate increased ACTH secretion suggesting that increased cortisol secretion is a consequence of increased ACTH secretion. The mean cortisol to ACTH ratio is also not significantly different between controls and depressed patients, leading us to conclude that there is no clear dissociation between the $\mathrm{ACTH}$ and cortisol secretion.

We also examined circadian and ultradian rhythms in cortisol secretion. We observed no difference between groups in the time of the ACTH or cortisol maxima or amplitude. Using the definition of either Van Cauter et al. (1996) or Linkowski et al. (1985) to define the onset and offset of the cortisol quiescent period, we observed no differences in the onset time, offset time or the quiescent period length in depressed women. This is in contrast with our studies using metyrapone administration restricted to the evening, where we observed clear evidence of activation of the HPA axis in depressed individuals (Young et al. 1994).

Both ACTH and cortisol are secreted in a pulsatile fashion. These ACTH and cortisol pulses are believed to result from pulses of CRH and thus represent an intrinsic central nervous system pulse generator, analogous to the pulse generator demonstrated for GnRH secretion. Our pulsatility analysis of cortisol secretion demonstrated pulses throughout the $24 \mathrm{hr}$ with no evidence of changes in pulse frequency over the course of $24 \mathrm{hr}$. In fact, we continued to observe cortisol pulses throughout the quiescent period, suggesting that $\mathrm{CRH}$ continues to be secreted throughout the $24 \mathrm{hr}$, but that pulse amplitude variation contributes to the circadian rhythm of both hormones. In addition, it was difficult

Table 4. Circadian Analyses of ACTH and Cortisol

\begin{tabular}{|c|c|c|c|c|c|c|}
\hline & \multicolumn{3}{|c|}{ АСТН } & \multicolumn{3}{|c|}{ Cortisol } \\
\hline & $\begin{array}{c}\text { Maximum Time } \\
( \pm S E)\end{array}$ & $\begin{array}{c}\text { Minimum Time } \\
( \pm S E)\end{array}$ & $\begin{array}{c}\text { Amplitude } \\
( \pm S E)\end{array}$ & $\begin{array}{c}\text { Maximum Time } \\
( \pm S E)\end{array}$ & $\begin{array}{c}\text { Minimum Time } \\
( \pm S E)\end{array}$ & $\begin{array}{l}\text { Amplitude } \\
( \pm S E)\end{array}$ \\
\hline Controls & $7: 13 \pm 36 \mathrm{~min}$ & $22: 39 \pm 31 \mathrm{~min}$ & $5.19 \pm 0.43$ & $7: 07 \pm 25 \mathrm{~min}$ & $23: 35 \pm 29 \mathrm{~min}$ & $12.11 \pm 0.63$ \\
\hline Patients & $7: 42 \pm 23 \mathrm{~min}$ & $23: 29 \pm 40 \mathrm{~min}$ & $5.3 \pm 0.39$ & $8: 11 \pm 25 \mathrm{~min}$ & $23: 28 \pm 21 \mathrm{~min}$ & $12.92 \pm 0.84$ \\
\hline
\end{tabular}


to account for the change in cortisol and ACTH secretion across the circadian rhythm as resulting from pulsatile cortisol secretion only, resulting in a need to involve more than one type of cortisol secretory activity, both baseline and pulsatile components.

Several other studies have reported on ACTH pulse frequency in either normal subjects or depressed patients. The number of pulses detected is dependent upon the pulse detection algorithm used. The older algorithms such as Cluster or Pulsar detect few pulses (10 or 6.4 pulses $/ 24 \mathrm{hr}$ ) than newer pulse detection algorithms such as deconvolution, which found 40 pulses in $24 \mathrm{hr}$. Our estimate of 23 and 26 secretory episodes per $24 \mathrm{hr}$ is intermediate between the number of pulses observed by Iranmanesh et al. (1990) with deconvolution and that detected by Cluster or Pulsar. Our data do not show any difference in patients and controls in pulse number. The reports of Mortola et al. (1987) and Deuschle et al. (1997) using Pulsar and Cluster show more pulses of ACTH in depressed subjects than normal controls. Because the older pulse detection algorithms failed to detect some of the ACTH pulse or secretory events, the greater number of ACTH pulses seen in depressed patients compared to controls in these reports may represent pulses that were easier to detect because of larger pulse amplitude in the depressed patients

Mortola et al. (1987) observed only five cortisol pulses $/ 24 \mathrm{hr}$, which is half the number of pulses they observed for ACTH, while Linkowski et al. (1985) found 17 pulses of cortisol, with no difference between patients and controls. Of the four studies examining cortisol pulsatility (including ours) only the report of Deuschle et al. (1997) report increased number of pulses. Deuschle et al. (1997) analyzed data over six hours and again used a pulse detection program that appears to underestimate pulse number. Thus, overall the evidence suggests that increased cortisol secretion is not due to increased pulse number but rather either increased amplitude of pulses or increased baseline cortisol not secreted in a pulsatile manner. This suggests that the CNS area controlling pulsatile hormone bursts (i.e., the pulse generator) is normal in depression.

In conclusion, our study suggests that increased cortisol secretion is found in only a small percent $(24 \%)$ of depressed premenopausal women. While as a group, women who met RDC criteria for definite endogenous showed elevated cortisol, the increase was not significant in this subgroup. Examining the six depressed women with the highest mean cortisol showed no clear association with diagnostic subgroup. Furthermore, women who met criteria for atypical depression showed normal cortisol. Both ACTH and cortisol appear to be elevated in the same subjects, suggesting a suprapituitary origin of the ACTH and cortisol hyperactivity. There were no differences between patients and controls in pulse number of either ACTH or corti- sol. Nor was there a difference in mean pulse amplitude. In fact, the increase in cortisol and ACTH appeared to be in the baseline component of secretion. Finally, we found no evidence of alterations in circadian or ultradian parameters of the HPA axis in depressed women. While surprising, the young age of the study population may account for the relatively normal HPA axis parameters, since age appears to be a significant factor in phase advancing the cortisol rhythm and also in increasing the rates of dexamethasone non-suppression (Akil et al. 1993; Halbreich et al. 1984; Oxenkrug et al. 1983; Van Cauter et al. 1996). Finally, the relatively small increases in cortisol observed in these depressed subjects may be enough to restrain the central overactivity, leading to a great underestimate of the degree of HPA axis activation and circadian disruption observed in these subjects. Newer studies examining the HPA axis in an open loop state (metyrapone) could shed further light on these issues.

\section{REFERENCES}

Akil H, Haskett R, Young EA, Grunhaus L, Kotun J, Weinberg V, Greden J, Watson SJ (1993): Multiple HPA profiles in endogenous depression: Effect of age and sex on cortisol and beta-endorphin. Biological Psychiatry 33:73-85

Belchetz PE, Plant TM, Nakai Y (1987): Hypophyseal responses to continuous and intermittent delivery of gonadotropin-releasing hormone. Science 202:631-633

Carroll BJ, Curtis GC, Mendels J (1976a): Neuroendocrine regulation in depression I. Limbic system-adrenocortical dysfunction. Arch Gen Psychiatry 33:1039-1044

Carroll BJ, Curtis GC, Mendels J (1976b): Neuroendocrine regulation in depression II. Discrimination of depressed from nondepressed patients. Arch Gen Psychiatry 33:1051-1058

Carroll BJ, Feinberg M, Greden JF, Tarika J, Albala AA, Haskett RF, James N, Kronfol Z, Lohr N, Steiner M, DeVigne JP, Young EA (1981): A specific laboratory test for the diagnosis of melancholia: Standardization, validation, and clinical utility. Arch Gen Psychiatry 38:15-22

Crofford LJ, Pillemer SR, Kalogeras KT, Cash JM, Michelson D, Kling MA, Sternberg EM, Gold PW, Chrousos GP, Wilder RL (1994): Hypothalamic-pituitary-adrenal axis perturbations in patients with fibromyalgia. Arthritis Rheumatism 37:1583-1592

Demitrack MA, Dale JK, Straus SE, Laue L, Liswak SJ, Kruesi MJ, Chrousos GP, Gold PW (1991): Evidence for impaired activation of the hypothalamic-pituitary-adrenal axis in patients with chronic fatigue syndrome. J Clin Endocrinology Metabolism 73:1224-1234

Deuschle M, Schweiger U, Weber B, Gotthardt U, Korner A, Schmider J, Standhardt H, Lammers C-H, Heuser I (1997): Diurnal activity and pulsatility of the hypothalamus-pituitary-adrenal system in male depressed 
patients and healthy controls. J Clin Endocrinology Metabolism 82:234-238

Guo W, Wang Y, Brown MB (1999): A signal Extraction approach to modeling hormone time series with pulses and a changing baseline. J Am Stat Association 94:746756

Halbreich U, Asnis GM, Schindledecker R, Zurnoff B, Nathan RS (1985): Cortisol secretion in endogenous depression I. Basal plasma levels. Arch Gen Psychiatry 42:909-914

Halbreich U, Asnis GM, Zumoff B, Nathan RS (1984): The effect of age and sex on cortisol secretion depressives and normals. Psychiatry Res 13:221-2229

Iranmanesh A, Lizarralde G, Short D, Veldhuis JD (1990): Intensive venous sampling paradigms disclose high frequency adrenocorticotropin release episodes in normal men. J Clin Endocrinol Metab 71:1276-83

Krishnan KRR, Ritchie JC, Saunders WB, Nemeroff CB, Carroll BJ (1990): Adrenocortical sensitivity to low-dose ACTH administration in depressed patients. Biol Psychiatry 27:930-933

Kushler RH, Brown MB (1991): A model for the identification of hormone pulses. Stat Med 10:329-340

Linkowski P, Mendelwicz J, LeClercq R, Brasseur M, Hubain P, Goldstein J, Copinschi G, van Cauter E (1985): The 24 hour profile of ACTH and cortisol in major depressive illness. J Clin Endocrinol Metab 61:429-438

Marshall JC, Dalkin AC, Haisenleder DJ, Paul SJ, Ortolano GA, Kelch RP (1991): Gonadotropin-releasing hormone pulses: Regulators of gonadotropin synthesis and ovulatory cycles. Recent Progress in Hormone Research 47:155-187

Mortola JF, Liu JH, Gillan JC, Rasmussen DD, Yen SSC (1987): Pulsatile Rhythms of ACTH and cortisol in women with endogenous depression: evidence for increased ACTH pulse frequency. J Clin Endocrinol Metab 65:962-968

Oxenkrug GF, Pomara N, McIntyre IM, Branconnier RJ, Stanley M, Gershon S (1983): Aging and cortisol resistance to suppression by dexamethasone: A positive correlation. Psychiatry Res 10:125-130

Pfohl B, Sherman B, Schlecte J, Stone R (1985): Pituitary/ adrenal axis rhythm disturbances in psychiatric patients. Arch Gen Psychiatry 42:897-903
Poland RE, Rubin RT, Lesser IM, Lane LA, Hart PJ (1987): Neuroendocrine aspects of primary endogenous depression. II. Serum dexamethasone concentrations and hypothalamic-pituitary-adrenal cortical activity as determinants of the dexamethasone suppression test response. Arch Gen Psychiatry 44:790-795

Rubin RT, Poland RE, Lesser IM, Winston RA, Blodgett N (1987): Neuroendocrine aspects of primary endogenous depression I. Cortisol secretory dynamics in patients and matched controls. Arch Gen Psychiatry 1987. 44:328-336

Sachar EJ, Hellman L, Roffwarg HP, Halpern FS, Fukush DK, Gallagher TF (1973): Disrupted 24 hour patterns of cortisol secretion in psychotic depressives. Arch Gen.Psychiatry 28:19-24

Tsigos C, Chrosous GP (1994): Physiology of the hypothalamic-pituitary-adrenal in health and dysregulation in psychiatric and autoimmune disorders. Endocrinology \& Metabolism Clinics of North America 23:451-466

Van Cauter E, Leproult R, Kupfer, DJ (1996): Effects of gender and age on the levels and circadian rhythmicity of plasma cortisol. J Clin Endocrinol Metab 81:2468-2473

Veldhuis JD, Johnson ML (1986): Cluster analysis: A simple versatile and robust algorithm for endocrine pulse detection. Amer J Physiol 250:E486-E493

Veldhuis JD, Johnson ML (1992): Deconvolution of hormone data. Methods in Enzymology 210:539-575

Wirz-Justice A (1994): Biological rhythms in affective disorders. In Kupfer FEB (ed), ACNP Fourth Generation of Progress. New York, Raven Press, pp 999-1017

Young EA, Lopez JF, Murphy-Weinberg V, Watson SJ, Akil $H$ (1997): Normal pituitary response to metyrapone in the morning in depressed patients: Implications for circadian regulation of CRH secretion. Biological Psychiatry 41:1149-1155

Young EA, Kotun J, Haskett RF, Grunhaus L, Greden JF, Watson SJ, Akil H (1993): Dissociation between pituitary and adrenal suppression to dexamethasone in depression. Arch Gen Psychiatry 50:395-403

Young EA, Haskett RF, Grunhaus L, Pande A, Murphy Weinberg V, Watson SJ, Akil H (1994): Increased circadian activation of the hypothalamic pituitary adrenal axis in depressed patients in the evening. Arch Gen Psychiatry 51:701-707 